\section{(6) OPEN ACCESS}

\title{
Cervical ectopy: associations with sexually transmitted infections and HIV. A cross-sectional study of high school students in rural South Africa
}

\author{
Elisabeth Kleppa, ${ }^{1,2}$ Sigve D Holmen, ${ }^{1,2}$ Kristine Lillebø, ${ }^{1,2}$ Eyrun F Kjetland, 1,3 \\ Svein Gunnar Gundersen, ${ }^{4,5}$ Myra Taylor, ${ }^{3}$ Prashini Moodley, ${ }^{6}$ Mathias Onsrud $^{7}$
}

- Additional material is published online only. To view please visit the journal online (http://dx.doi.org/10.1136/ sextrans-2014-051674).

For numbered affiliations see end of article.

\section{Correspondence to}

Dr Elisabeth Kleppa, Norwegian Centre for Imported and Tropical Diseases, Department of Infectious Diseases, Oslo University Hospital Ullevaal, Postboks 4956 Nydalen, Oslo 0424, Norway; elisabeth.kleppa@medisin.uio.no

EK and SDH contributed equally.

Received 28 May 2014 Revised 4 September 2014 Accepted 14 September 2014 Published Online First 3 October 2014

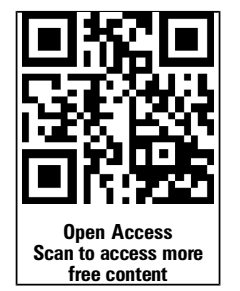

\section{ABSTRACT}

Objectives It has been hypothesised that ectopy may be associated with increased susceptibility to sexually transmitted infections (STIs). In this cross-sectional study, we wanted to explore the association between STIS (including HIV) and cervical ectopy.

Methods We included 700 sexually active young women attending randomly selected high schools in a rural district in KwaZulu-Natal, South Africa. The district is endemic of HIV and has a high prevalence of STIs. We did computer-assisted measurements of the ectocervical area covered by columnar epithelium (ectopy) in colposcopic images and STI analyses on cervicovaginal lavage and serum samples. All participating women answered a questionnaire about sexual behaviour and use of contraceptives.

Results The mean age was 19.1 years. Ectopy was found in $27.2 \%$, HIV in $27.8 \%$, chlamydia in $25.3 \%$ and gonorrhoea in $15.6 \%$. We found that age, parity, chlamydia and gonorrhoea, years since menarche, years since sexual debut and number of sexual partners were associated with ectopy. In multivariate analysis with chlamydia infection as the dependent variable, women with ectopy had increased odds of having chlamydia infection (adjusted OR 1.78, $p=0.033$ ). In women under 19 years of age, we found twofold higher odds of being HIV-positive for those with ectopy (OR 2.19, $p=0.014$ ).

Conclusions In conclusion, cervical ectopy is associated with Chlamydia trachomatis infection and HIV in the youngest women.

\section{INTRODUCTION}

Young women carry a disproportionally high risk of contracting sexually transmitted infections (STI) including HIV. ${ }^{1}{ }^{2}$ A complex relationship between sexual behaviour and biological risk factors contribute to this tendency. ${ }^{3}$ Cervical ectopy, the presence of columnar epithelium on the ectocervix, has been suggested to influence women's susceptibility to STIs. ${ }^{4}$

Ectopy occurs when the columnar epithelium, normally found in the endocervical channel, extends onto the ectocervix. This epithelium consists of a single layer of columnar cells in contrast with the stratified (multilayer) squamous epithelium covering the rest of the ectocervical surface. ${ }^{5}$ Ectopy is commonly seen in young women. ${ }^{5} 6$ During and after puberty, the process of metaplasia transforms the columnar epithelium into squamous epithelium and the cervical ectopy decreases. ${ }^{5}$
Epidemiological studies have found an association between cervical ectopy and infection with Chlamydia trachomatis. ${ }^{4-9}$ This intracellular bacterium resides in columnar epithelial cells, making cervical ectopy a favourable condition for infection. ${ }^{7}$ It has been hypothesised that hormonal contraceptives may further enhance this susceptibility, as they have been shown to be associated with both increased ectopy and chlamydia infection..$^{9-11}$ Several studies have also found an association between ectopy and HIV infection, but the topic remains controversial. ${ }^{461213}$ Female genital schistosomiasis (FGS) has been shown to be associated with HIV in cross-sectional studies, ${ }^{14}$ but to our knowledge, there have been no studies on the association with cervical ectopy. The ectocervical area covered by columnar epithelium is usually estimated subjectively by the investigating clinician, whereas computer assisted measurement of the area on colposcopic images has been shown to be a more reliable method. ${ }^{15} 16$

In this study of young women, we investigated the relationship between objectively measured ectopy and common reproductive tract infections. Due to the cross-sectional design of the study we could not explore causality. However, it seems biologically plausible that cervical ectopy may be associated with increased susceptibility to STIs. To our knowledge, this is the first study investigating ectopy in young women in an area with high prevalence of STIs including HIV.

\section{MATERIALS AND METHODS \\ Enrolment}

The participants were recruited in 2010-2012 from an ongoing study in coastal KwaZulu-Natal, South Africa, focusing on various aspects of FGS and STIs. Of the women $(n=1074$, figure 1$)$ who visited the research clinic during this time period, all who had an image taken during the gynaecological exam that met the inclusion criteria $(n=700)$ were included in this cross-sectional study. Young women from 31 randomly selected high schools were invited. All included women were sexually active and in their final years of high school. Women were not recruited based on symptoms.

The rural district on the east coast is one of the poorest in South Africa, endemic for urogenital schistosomiasis and has a high prevalence of HIV, chlamydia, gonorrhoea and trichomoniasis. 


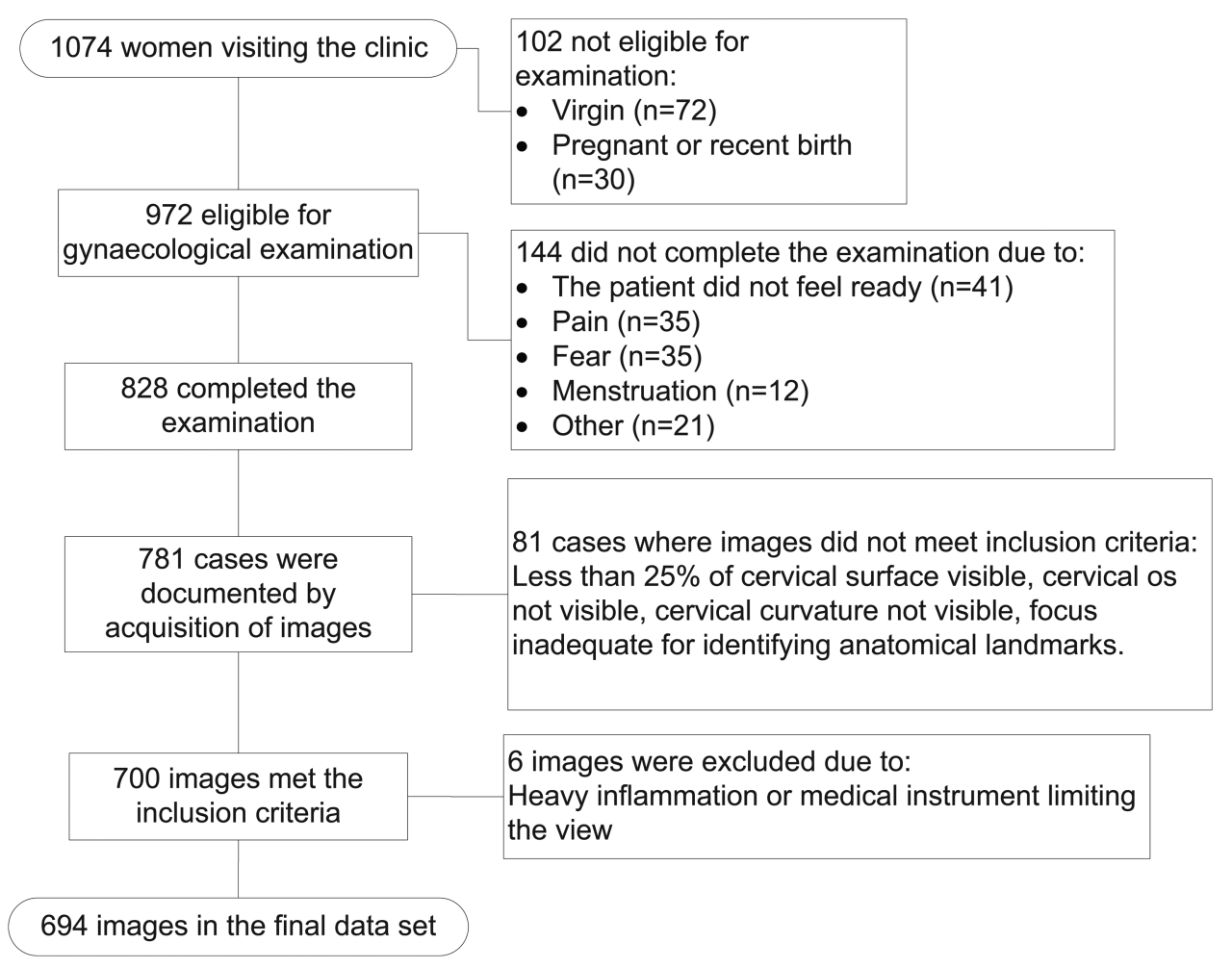

Figure 1 Flowchart showing inclusion of images.

\section{Clinical investigation and questionnaire}

The study participants were interviewed by trained research assistants. The questionnaire was in the local language (isiZulu) and included questions on behavioural factors (including sexual behaviour, alcohol and drug use), contraceptives, obstetric history and urogenital symptoms.

Gynaecological examinations with colposcopy were performed by two female medical doctors (EK and KL). Pregnant women were not investigated. Cervicovaginal lavage (CVL) samples were collected by spraying $10 \mathrm{~mL}$ of saline on the cervix four times before drawing it back into a syringe and depositing it into tubes for frozen storage. Blood was collected into sterile acid-citrate-dextrose anticoagulated Vacutainer tubes. The examinations were documented using an Olympus OCS 500 colposcope with a mounted Olympus E 42010 megapixel (Mpx) single lens reflex (SLR) device, or a Leisegang colposcope with a Canon EOS 40D $10 \mathrm{Mpx}$ SLR. The image files were stored using high-quality JPEG compression along with data from the clinical investigation.

\section{Image material}

During the inclusion period, a total of 781 women underwent gynaecological examination with acquisition of colposcopic images. One image was selected per patient based on the following inclusion criteria: at least $25 \%$ of the ectocervical surface was visible, the cervical os was visible, a part of the cervical curvature was visible, and the focus was adequate for identifying anatomical landmarks. During the measurements, images were excluded if there was heavy inflammation rendering the evaluation impossible or if there was a non-cervical element rendering the extent of ectopy non-evaluable (such as blood, discharge or a medical instrument). The best image was chosen independent of whether it was taken before or after sampling for Pap smear, cervico-vaginal lavage or application of acetic acid.

\section{Computer assisted measurement of cervical ectopy}

All measurements were performed using the open source image analysis software Image (US National Institutes of Health). It allows for a visually guided delimitation of structures and a subsequent measurement of the selected area (figure 2). We further calculated the ectopy as a fraction of the total

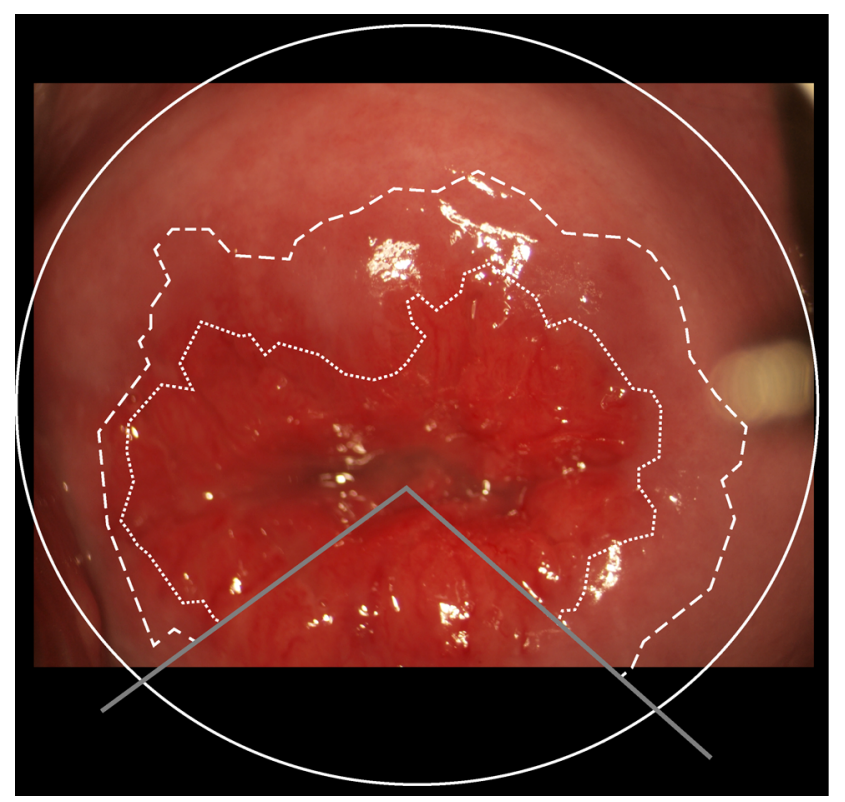

Figure 2 A photocolposcopic image of the ectocervix. Solid line: Delimiting the cervical area. Dashed line: Original squamocolumnar junction, outer delimitation of the transformation zone. Dotted line: Current squamocolumnar junction, represents the border between columnar epithelium and metaplastic squamous epithelium in the transformation zone. 
ectocervical area. Finally, the images were saved with the measurement overlays for verification and adjustment by a senior gynaecologist (MO).

The ectocervical area was delimited by using the cervical os as the centre of an elliptical shape that was manually fitted to conform to the visible cervical boundaries. The original squamocolumnar junction was defined as the outer delimitation of any of the following structures: glandular openings, Nabothian cysts and primary rugae extending from the cervical os. In cases where such anatomical landmarks were absent or too sparse, the original squamocolumnar junction was not measured. Finally, the current squamocolumnar junction (confining the area of ectopy) was defined as a distinct transition of colour from deep red to brighter red: The columnar epithelium is single layered and appears dense red whereas the transformation zone is covered in squamous epithelium, appearing brighter in colour.

In cases where the original or current squamocolumnar junctions were partially masked by medical instruments, blood or as a result of the angle of inspection or photographic section, the analysis of the ectocervix was divided into sectors. The largest sector(s) in which it was possible to demarcate the squamocolumnar junction was used as a basis for estimating the squamocolumnar junction that was impossible to measure.

The output of the measurements was in numbers of squared pixels. The focal distance of each image was unknown. In consequence, it was impossible to convert the computer measurements into absolute values of squared millimetres. All measurements were, therefore, converted to fractions of the total ectocervical area, thus representing relative values. In previous studies, a cut-off level of $10 \%$ of the cervical area covered by columnar epithelium has been used to define a cervix as having ectopy. ${ }^{6} 12$ The investigators were blinded to the patients' STI results and questionnaire information.

\section{Laboratory analyses}

CVL samples were analysed by strand displacement assay for Neisseria gonorrhoeae and C. trachomatis (ProbeTec CT/GT, Becton, Dickinson and Company (BD), Franklin Lakes, New Jersey, USA). A Gram stain was made from the CVL and scored for bacterial vaginosis using Nugent's scoring methodology. PCR was used to screen for herpes simplex virus types 1 and 2 (HSV 1 and 2) and Trichomonas vaginalis (in-house PCR, Laboratory of Infection, Prevention and Control, UKZN, Durban, South Africa). ${ }^{17} 18$ Syphilis serology was performed using rapid plasma reagin (Macro Vue test 110/112, BD) and verification was done using Treponema pallidum hemagglutination assay (Omega diagnostics Group PLC, Alva, Scotland, UK). HIV serology was done using rapid test kits (Bioline Rapid Test HIV, New Jersey, USA and Sensa Tri-Line HIV Test Kit, Durban, South Africa). All serology was done on serum samples frozen at $-80^{\circ} \mathrm{C}$.

\section{Ethics}

The study was approved by the Biomedical Research Ethics Administration (BREC), University of KwaZulu-Natal, South Africa 20 February 2009 (Ref BF029/07, renewed yearly) and by the Provincial Department of Health, Pietermaritzburg, South Africa on 3 February 2009 (Reference HRKM010-08). The Regional Committee for Medical and Health Research Ethics (REC) South Eastern Norway gave ethical clearance on 17 September 2007 (Ref 469-07066a1.2007.535). The Departments of Health and Education in Ugu district, KwaZulu-Natal also gave permissions.
Only sexually active women were invited. All participants signed individual, written consent forms. The ethical committees, BREC and REC, were aware that 15 -year-olds to 17-year-olds were participating in the study and specifically approved the consent procedure (independent minor consent, no parental consent). According to South African legislation, persons over the age of 12 years could consent independently to participate in research. Those who presented with symptoms, signs or tested positive on subsequent laboratory analyses of STIs were offered treatment in accordance with a South African syndromic protocol. Partner treatment was also offered. HIV testing and follow-up was done in accordance with South African guidelines. HIV positive patients were referred to local clinics for follow-up and antiretroviral treatment. Anti-schistosomal treatment was offered to all.

\section{Statistical analyses}

Statistical analyses were performed using IBM SPSS Statistics V.20 (SPSS, Armonk, New York, USA). Pearson's $\chi^{2}$ test and Fisher's exact test were used to compare the characteristics of women with and without ectopy. Univariate and multivariate logistic regression models were constructed to analyse the associations between ectopy, age, parity and gynaecological pathology. A significance level of 5\% was used throughout.

\section{RESULTS}

Out of 781 participants, 694 (88.9\%) could be included in the evaluation (figure 1). Mean age was 19.1 years and followed a near normal distribution (SD 2.1, range 15-31). Half of the women had given birth $(383 / 682,56.2 \%)$. Parous women had a mean of 1.2 pregnancies (SD 0.6) and their mean age was 19.8 years (SD 2.0). Injectable contraceptives were used by $30.0 \%$ of the women (204/681), while only $1.5 \%$ (10/680) used contraceptive pills. At least one STI (not including HIV) was found in 306 women (60.6\%). The HIV prevalence was 27.8\% (159/ 572). Only 9.1\% (62/681) reported that they knew they were HIV positive, of which $23.0 \%(14 / 61)$ reported to be on antiretroviral treatment (one did not answer).

\section{Cervical ectopy}

Ectopy, measured as a fraction of the total ectocervical area, did not follow a normal distribution. The median degree of ectopy was $4.0 \%$ (range $0.0 \%-69.5 \%$ ). Using a $10 \%$ cut-off in defining ectopy, $27.2 \%(n=189)$ of the women had ectopy (table 1 , Web reference 1). Ectopy was associated with having been pregnant (OR 3.11, 95\% CI (2.14 to 4.52), p value <0.001). The level of ectopy was also increasing with age $(0.5 \%$ per year, $\mathrm{p}=0.019$ ). However, when adjusted for having given birth, no age-dependent increase was found $(p=0.83)$. The same tendency was found for years since menarche, years since sexual debut and number of lifetime sexual partners, which were significantly associated to degree of ectopy but not when adjusted for having given birth.

\section{Ectopy and C. trachomatis infection}

Of the women with ectopy, $35.0 \%$ tested positive for genital chlamydia infection as opposed to $21.6 \%$ of the women without ectopy (OR 1.96, 95\% CI (1.31 to 2.93), $\mathrm{p}=0.001$ ). Increasing ratio of ectopy was associated with an increasing OR of having chlamydia infection (figure 3). Women with more than $40 \%$ ectopy very often had chlamydia infection compared with women without ectopy (OR 5.08, 95\% CI (2.18 to 11.83), $\mathrm{p}<0.001)$. 
Table 1 Characteristics and test results of the study population comparing the group with cervical ectopy ( $\geq 10 \%$ of the area) to those without ectopy

\begin{tabular}{|c|c|c|c|c|c|}
\hline & & Ectopy $(\%)^{*}$ & No ectopy $(\%)^{*}$ & OR $(95 \% \mathrm{CI})$ & p Valuet \\
\hline \multicolumn{6}{|l|}{ Characteristics } \\
\hline Total & & $189(100)$ & $505(100)$ & & \\
\hline Age & $<19$ & $81 / 188(43)$ & $276 / 496(56)$ & 0.60 (0.43 to 0.84$)$ & 0.003 \\
\hline Never been pregnant & & $47 / 187(25)$ & 252/495 (51) & & \\
\hline Pregnant at least once & & 141/187 (75) & 243/495 (49) & 3.11 (2.14 to 4.52$)$ & $<0.001$ \\
\hline Years since menarche & $\begin{array}{l}0-4 \\
5-6 \\
\geq 7\end{array}$ & $\begin{array}{l}66(35) \\
65(35) \\
57(30)\end{array}$ & $\begin{array}{l}210(43) \\
167(34) \\
115(23)\end{array}$ & $\begin{array}{l}1.24(0.83 \text { to } 1.84) \\
1.58(1.04 \text { to } 2.40)\end{array}$ & $\begin{array}{l}0.293 \\
0.034\end{array}$ \\
\hline \multicolumn{6}{|l|}{ Infections } \\
\hline Chlamydia trachomatis & & $55 / 157(35)$ & $89 / 412(22)$ & 1.96 (1.31 to 2.93$)$ & 0.001 \\
\hline HIV & & $53 / 165(32)$ & 106/407 (26) & $1.34(0.91$ to 1.99$)$ & 0.142 \\
\hline Herpes simplex virus & & $6 / 158(4)$ & $26 / 408(6)$ & $0.58(0.23$ to 1.44$)$ & 0.239 \\
\hline Syphilis & & $6 / 147(4)$ & $9 / 344(3)$ & $1.58(0.55$ to 4.53$)$ & 0.388 \\
\hline Trichomonas vaginalis & & $40 / 170(24)$ & $87 / 424(21)$ & 1.19 (0.78 to 1.82$)$ & 0.419 \\
\hline Neisseria gonorrhoeae & & $38 / 156(24)$ & $70 / 412$ (17) & 1.57 (1.01 to 2.46$)$ & 0.046 \\
\hline Bacterial vaginosis & & $75 / 149(50)$ & 217/394 (55) & $0.83(0.57$ to 1.21$)$ & 0.323 \\
\hline Any sexually transmitted infection $\ddagger$ & & $102 / 150(68)$ & 204/355 (58) & 1.57 (1.05 to 2.35$)$ & 0.027 \\
\hline Grainy sandy patch§ & & 28/189 (15) & $70 / 505$ (14) & $1.08(0.67$ to 1.74$)$ & 0.748 \\
\hline \multicolumn{6}{|l|}{ Contraceptives } \\
\hline Contraceptive injection & & $61 / 187(33)$ & $143 / 494(29)$ & $1.19(0.83$ to 1.71$)$ & 0.350 \\
\hline Contraceptive pills & & $5 / 187(3)$ & $5 / 493(1)$ & $2.68(0.77$ to 9.37$)$ & 0.1489 \\
\hline Condom use (last intercourse) & & $64 / 140(46)$ & $158 / 380(42)$ & $1.18(0.80$ to 1.75$)$ & 0.398 \\
\hline \multicolumn{6}{|l|}{ Behavioural factors } \\
\hline Number of lifetime partners & $\geq 3$ & $41 / 186(22)$ & $147 / 492(30)$ & 0.66 (0.45 to 0.99$)$ & 0.042 \\
\hline Alcohol, age at debut ${ }^{* *}$ & $\begin{array}{l}\leq 15 \\
>15\end{array}$ & $\begin{array}{l}24 / 187(13) \\
83 / 187(44)\end{array}$ & $\begin{array}{r}82 / 491(17) \\
229 / 491(47)\end{array}$ & $\begin{array}{l}0.66(0.39 \text { to } 1.11) \\
0.82(0.57 \text { to } 1.17)\end{array}$ & $\begin{array}{l}0.119 \\
0.272\end{array}$ \\
\hline Drugs (ever used) & & $11 / 188(6)$ & $51 / 494(10)$ & $0.54(0.28$ to 1.06$)$ & 0.069 \\
\hline Years since sexual debut & $\geq 3$ & 95/187 (51) & 183/492 (37) & 1.74 (1.24 to 2.45$)$ & 0.001 \\
\hline Age at sexual debut & $\begin{array}{l}\leq 15 \\
16 \\
\geq 17\end{array}$ & $\begin{array}{l}52(28) \\
49(26) \\
86(46)\end{array}$ & $\begin{array}{l}112(23) \\
167(34) \\
215(44)\end{array}$ & $\begin{array}{l}0.63(0.40 \text { to } 1.00) \\
0.86(0.57 \text { to } 1.30)\end{array}$ & $\begin{array}{l}0.049 \\
0.480\end{array}$ \\
\hline Days since last intercourse & $\leq 7$ & $53 / 186(28)$ & $135 / 488(28)$ & 0.96 (0.66 to 1.40$)$ & 0.830 \\
\hline Anal sex & & $8 / 187(4)$ & $13 / 494(3)$ & 1.65 (0.67 to 4.06$)$ & 0.267 \\
\hline Forced sex & & 20/188 (11) & $45 / 494(9)$ & 1.19 (0.68 to 2.07$)$ & 0.543 \\
\hline
\end{tabular}

We fitted a multivariate logistic regression model with chlamydia as the dependent variable, where we adjusted for other STIs, age, condom use, number of partners and age at sexual debut. We found that women with ectopy had almost twofold increased odds of having chlamydia (adjusted OR (AOR) 1.78, 95\% CI (1.05 to 3.01), $\mathrm{p}=0.033)$.

\section{Ectopy and $N$. gonorrhoeae infection}

Gonorrhoea was found more frequently in the ectopy group $(24.4 \%)$ than in the group without ectopy $(17.0 \%$, OR 1.57 , 95\% CI (1.01 to 2.46), $\mathrm{p}=0.046$ ). None of the other STIs nor FGS was significantly associated with ectopy in univariate analysis (table 1). However, when adjusting for other STIs in a multivariate regression model, gonorrhoea was not significantly associated with ectopy (AOR 1.15, 95\% CI (0.70 to 1.88), $\mathrm{p}=0.578$.

\section{Ectopy and HIV infection}

For women under 19 years of age $(n=282)$, we found an HIV prevalence of $31.8 \%$ in women with ectopy as opposed to
$17.6 \%$ in women without ectopy (OR 2.19, 95\% CI (1.17 to 4.09), $\mathrm{p}=0.014$, Web reference 1). There was no significant association between cervical ectopy and HIV in women over 19 years of age $(\mathrm{p}=0.452)$.

\section{DISCUSSION}

In this study, we found a twofold increase of the odds of being HIV positive for the youngest women with ectopy. Furthermore, we found that chlamydia infection was independently associated with ectopy.

A large, longitudinal study conducted in South Africa in 2006 determined that ectopy was a risk factor for HIV. ${ }^{13}$ They found a weak association, and only for ectopy extending over $20 \%$ of the cervical surface. The women were, however, participants of a screening programme for cervical cancer and older than in our study ( $>35$ years). We observed a difference in the association between ectopy and HIV infection between age groups. It has previously been reported that young age is an independent risk factor for acquisition of HIV. ${ }^{1}$ It is possible that the influence of various HIV risk factors change during adolescence. 


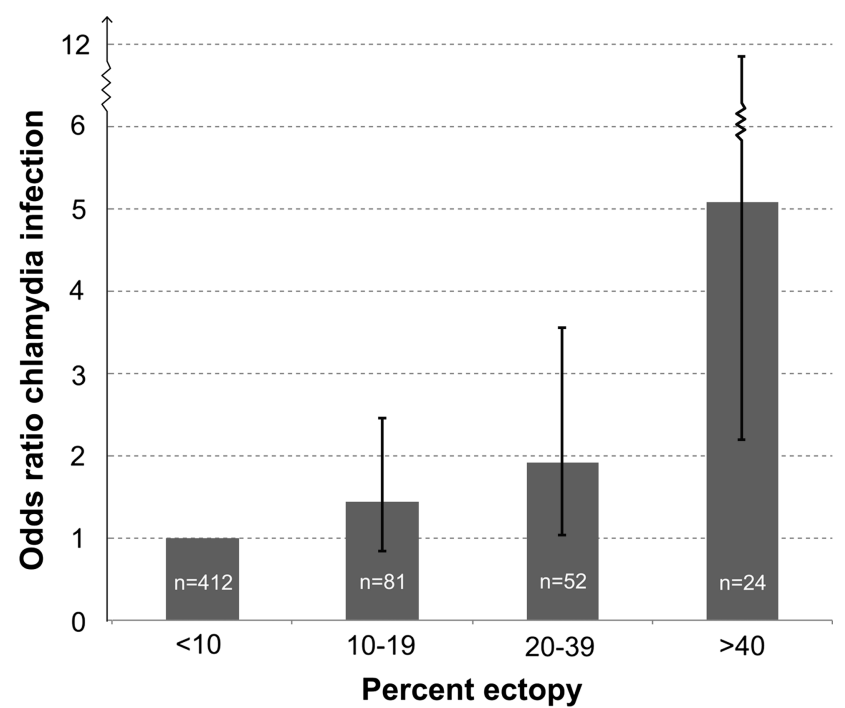

Figure 3 Graph showing the OR, with $95 \%$ Cls, of having Chlamydia trachomatis infection (y-axis) with increasing levels of cervical ectopy (x-axis). Participants without ectopy $(<10 \%)$ serve as the reference group.

Stratifying by age might help decipher risk factors in different age groups. A challenge is the timing of HIV acquisition in relation to the ectopy, as there is a natural decrease of ectopy over time. ${ }^{4}$ The youngest women in this population are likely to have HIV seroconverted more recently and may therefore give a better indication of risk factors present at the time of HIV acquisition. The single-layered columnar epithelium may represent a weaker physical barrier than the stratified squamous epithelium, leaving a woman with ectopy at higher risk of HIV infection. ${ }^{4}$ Furthermore, HIV target cells have been found in greater abundance in columnar than in squamous epithelium. ${ }^{4}$ Higher levels of HIV viral shedding in the genital tract have also been associated with ectopy. ${ }^{20}$

In accordance with other studies, we also found a strong association between ectopy and chlamydia infection. ${ }^{7} 9^{11}$ Due to the cross-sectional design of this study, we cannot determine the cause-effect relationship of the association. One longitudinal study reported increased risk of C. trachomatis acquisition when ectopy was present. $^{9}$ It seems biologically plausible that a larger area of columnar epithelium exposed on the ectocervical surface may increase the risk of infection. Our data indicate a doseresponse relationship between ectopy and chlamydia infection (figure 3). In the univariate analysis, both gonorrhoea and chlamydia infection was associated with ectopy. It has been suggested that chlamydia shedding could be increased with concurrent N. gonorrhoeae infection. ${ }^{21}$ Both these STIs may lead to serious consequences for women and increased risk of HIV acquisition. ${ }^{22}$ We did not find a significant association between ectopy and syphilis, T. vaginalis, bacterial vaginosis, HSV or FGS in univariate analysis (table 1).

Our finding that parity was associated with ectopy is in accordance with previous studies. ${ }^{10} \quad 12 \quad 23 \quad 24$ This can be related to normal changes of the cervix caused by the delivery or hormonal changes during pregnancy. This finding may indicate that parity could influence a woman's susceptibility to STIs. The number of oral contraceptive users was too low to draw any conclusion on the association with ectopy, but contraceptive injections of progestogen were not associated with ectopy.

\section{Limitations}

The size of the exposed columnar epithelium might depend on the opening of the speculum. Parous women have a wider and more open cervix and as a result, the ectopy could be overestimated. Even though the columnar epithelium is generally easy to distinguish from the surrounding squamous epithelium, we cannot rule out the possibility that some areas of immature metaplasia, inflammation or otherwise hyperaemic epithelium could mimic ectopy and hence lead to an overestimation of the area. In cases of doubt, the use of acetic acid was helpful.

The planimetric measuring tool provides relative numbers as a fraction of the total ectocervical area. Although this gives an indication of the degree of ectopy, which allows for comparison with other subjects, it may be of more biological importance to measure the absolute area of ectopy. This is only possible if the exact depth of field is known, but this information could not be extracted from our images.

T. vaginalis, N. gonorrhoeae, C. trachomatis and HSV were analysed in vaginal lavage samples. Similar methods have been used in previous studies and have been validated for HSV and T. vaginalis. ${ }^{17} 1825$

Our analysis of N. gonorrhoeae and C. trachomatis in lavage showed a similar ratio between the two infections compared to previous reports from South Africa. ${ }^{26} 27$ Furthermore, the DNA-based detection technique that was used for N. gonorrhoeae and C. trachomatis is reported to have close to $100 \%$ specificity. $^{28}$

We did not collect information about the practice of vaginal douching, which may be negatively associated with cervical ectopy. ${ }^{11}$

\section{Strengths}

The study population was extensively explored for factors that have previously been hypothesised to influence ectopy. As our study participants were recruited from schools regardless of symptoms, the participants were more likely representative of the general population at this age than women attending health facilities.

This study has the advantage that ectopy area calculations were objective. Computer assisted planimetry on digital, colposcopic images offered a precise and reproducible way of measuring the relative area of ectopy on the cervix. The investigation was performed at a point in time where there are almost no adult survivors of mother-to-child HIV transmission, hence almost all HIV infections were likely to have been sexually transmitted. In this young population, it is therefore probable that STIs including HIV infections occurred relatively recent to our measurement of ectopy. As the degree of ectopy changes over time, this temporal proximity of measurement is a clear advantage.

\section{CLINICAL RELEVANCE AND CONCLUSION}

STIs and especially HIV have serious consequences for women. The high prevalence of STIs including HIV in this group of young women is worrying and warrants further studies. Thorough knowledge about possible risk factors is needed. Ectopy may be a biological risk factor for chlamydia infection and for HIV in the young women. Treatment of ectopy by methods like cryocauterisation and electrocoagulation has been discussed, but the effect is still uncertain. ${ }^{29}$ Counselling on barrier contraceptives might be of special importance when cervical ectopy is found in young women at risk. 


\section{Key messages}

- Columnar epithelium found on the ectocervical surface is called ectopy and it can be a normal finding in young women.

- Young women with ectopy were found to have more Chlamydia trachomatis, Neisseria gonorrhoeae and HIV infections.

- Ectopy may render the woman more susceptible to sexually transmitted infections.

\section{Author affiliations}

${ }^{1}$ Norwegian Centre for Imported and Tropical Diseases, Department of Infectious Diseases, Oslo University Hospital Ullevaal, Oslo, Norway

${ }^{2}$ Faculty of Medicine, University of Oslo, Oslo, Norway

${ }^{3}$ School of Public Health Medicine, Nelson R Mandela School of Medicine, University of KwaZulu-Natal (UKZN), Durban, South Africa

${ }^{4}$ Research Unit, Sorlandet Hospital, Kristiansand, Norway

${ }^{5}$ Department of Global Development and Planning, University of Agder, Kristiansand, Norway

${ }^{6}$ Department of Infection Prevention and Control, School of Laboratory Medicine and Medical Sciences, Nelson R Mandela School of Medicine, UKZN, Durban, South Africa

${ }^{7}$ Department of Gynecology, OUH, Norway

\section{Handling editor Jackie A Cassell}

Acknowledgements Thanks to the local staff, professors Bjørn Myrvang and Leiv Sandvik.

Contributors EFK, SGG, MO, MT, EK and SDH contributed to the conception and design of the work. Acquisition, analysis or interpretation of the data was done by $E K, S D H, K L, P M$ and MO. EK and SDH drafted the first version of the paper, and $\mathrm{KL}$, EFK, SGG, MT, PM and MO revised the draft. All the co-authors approved the final version of the manuscript.

Funding The research project was funded by grants from the South-Eastern Norway Regional Health Authority and European Research Council under the European Union's Seventh Framework Programme.

\section{Competing interests None.}

Ethics approval Biomedical Research Ethics Administration, University of KwaZulu-Natal, South Africa and by the Provincial Department of Health, Pietermaritzburg, South Africa and The Regional Committee for Medical and Health Research Ethics (REC) South Eastern Norway.

Provenance and peer review Not commissioned; externally peer reviewed.

Open Access This is an Open Access article distributed in accordance with the Creative Commons Attribution Non Commercial (CC BY-NC 4.0) license, which permits others to distribute, remix, adapt, build upon this work non-commercially, and license their derivative works on different terms, provided the original work is properly cited and the use is non-commercial. See: http://creativecommons.org/ licenses/by-nc/4.0/

\section{REFERENCES}

1 Sarkar K, Bal B, Mukherjee R, et al. Young age is a risk factor for HIV among female sex workers - an experience from India. J Infect 2006;53:255-9.

2 Ramjee G, Wand H. Geographical clustering of high risk sexual behaviors in "hot-spots" for HIV and sexually transmitted infections in Kwazulu-Natal, South Africa. AIDS Behav 2014:18:317-22.

3 Yi TJ, Shannon B, Prodger J, et al. Genital immunology and HIV susceptibility in young women. Am J Reprod Immunol 2013;69(Suppl 1):74-9.
4 Venkatesh KK, Cu-Uvin S. Assessing the relationship between cervical ectopy and HIV susceptibility: implications for HIV prevention in women. Am J Reprod Immunol 2013;69(Suppl 1):68-73.

5 Jacobson DL, Peralta L, Graham NM, et al. Histologic development of cervical ectopy: relationship to reproductive hormones. Sex Transm Dis 2000;27:252-8.

6 Moscicki a B, Ma Y, Holland C, et al. Cervical ectopy in adolescent girls with and without human immunodeficiency virus infection. J Infect Dis 2001;183:865-70.

7 Lee V, Tobin JM, Foley E. Relationship of cervical ectopy to chlamydia infection in young women. J Fam Plann Reprod Health Care 2006;32:104-6.

8 Jacobson DL, Peralta L, Farmer $\mathrm{M}$, et al. Relationship of hormonal contraception and cervical ectopy as measured by computerized planimetry to chlamydial infection in adolescents. Sex Transm Dis 2000;27:313-19.

9 Morrison CS, Bright P, Wong EL, et al. Hormonal contraceptive use, cervical ectopy, and the acquisition of cervical infections. Sex Transm Dis 2004;31:561-7.

10 Bright PL, Norris Turner A, Morrison CS, et al. Hormonal contraception and area of cervical ectopy: a longitudinal assessment. Contraception 2011;84:512-19.

11 Critchlow CW, Wölner-Hanssen P, Eschenbach DA, et al. Determinants of cervical ectopia and of cervicitis: age, oral contraception, specific cervical infection, smoking, and douching. Am J Obstet Gynecol 1995;173:534-43.

12 Moss GBG, Clemetson D, D'Costa L, et al. Association of cervical ectopy with heterosexual transmission of human immunodeficiency virus: results of a study of couples in Nairobi, Kenya. J Infect Dis 1991;164:588-91.

13 Myer L, Wright TC, Denny L, et al. Nested case-control study of cervical mucosal lesions, ectopy, and incident HIV infection among women in Cape Town, South Africa. Sex Transm Dis 2006;33:683-7.

14 Kjetland EF, Leutscher PD, Ndhlovu PD. A review of female genital schistosomiasis. Trends Parasitol 2012;28:58-65.

15 Gilmour E, Ellerbrock TV, Koulos JP, et al. Measuring cervical ectopy: direct visual assessment versus computerized planimetry. Am J Obstet Gynecol 1997;176(1 Pt 1):108-11.

16 Morrison CS, Bright P, Blumenthal PD, et al. Computerized planimetry versus clinical assessment for the measurement of cervical ectopia. Am J Obstet Gynecol 2001;184:1170-6.

17 Pillay A, Radebe F, Fehler G, et al. Comparison of a TaqMan-based real-time polymerase chain reaction with conventional tests for the detection of Trichomonas vaginalis. Sex Transm Infect 2007;83:126-9.

18 Aumakhan B, Hardick A, Quinn TC, et al. Genital herpes evaluation by quantitative TaqMan PCR: correlating single detection and quantity of HSV-2 DNA in cervicovaginal lavage fluids with cross-sectional and longitudinal clinical data. Virol J 2010;7:328.

19 Kjetland EF, Ndhlovu PD, Gomo E, et al. Association between genital schistosomiasis and HIV in rural Zimbabwean women. AIDS 2006;20:593-600.

20 Homans J, Christensen S, Stiller T, et al. Permissive and protective factors associated with presence, level, and longitudinal pattern of cervicovaginal HIV shedding. J Acquir Immune Defic Syndr 2012;60:99-110

21 Barnes RC, Katz BP, Rolfs RT, et al. Quantitative culture of endocervical Chlamydia trachomatis. J Clin Microbiol 1990;28:774-80.

22 Ward H, Ronn M. Contribution of sexually transmitted infections to the sexual transmission of HIV. Curr Opin HIV AIDS 2010;5:305-10.

23 Jacobson $\mathrm{DL}$, Peralta $\mathrm{L}$, Farmer $\mathrm{M}$, et al. Cervical ectopy and the transformation zone measured by computerized planimetry in adolescents. Int J Gynaecol Obstet 1999:66:7-17.

24 Louv WC, Austin H, Perlman J, et al. Oral contraceptive use and the risk of chlamydial and gonococcal infections. Am J Obstet Gynecol 1989;160:396-402.

25 Kjetland EF, Gwanzura L, Ndhlovu PD, et al. Herpes simplex virus type 2 prevalence of epidemic proportions in rural Zimbabwean women: association with other sexually transmitted infections. Arch Gynecol Obstet 2005;272:67-73.

26 Wilkinson D, Abdool Karim SS, Harrison A, et al. Unrecognized sexually transmitted infections in rural South African women: a hidden epidemic. Bull World Heal Organ 1999;77:22-8.

27 Pettifor AE, Kleinschmidt I, Levin J, et al. A community-based study to examine the effect of a youth HIV prevention intervention on young people aged 15-24 in South Africa: results of the baseline survey. Trop Med Int Health 2005;10:971-80.

28 Fontana C, Favaro M, Cicchetti O, et al. Performance of strand displacement amplification assay in the detection of Chlamydia trachomatis and Neisseria gonorrhoeae. Jpn J Infect Dis 2005;58:283-8.

29 Machado Junior LC, Dalmaso ASW, Carvalho HB. Evidence for benefits from treating cervical ectopy: literature review. Sao Paulo Med J 2008;126:132-9. 